\title{
Pratiques
}

Linguistique, littérature, didactique

Conceptualisations de l'esprit critique, des formes de validation et de l'effacement énonciatif dans l'écriture universitaire en sciences infirmièreset dans les formations de sages-femmes

\section{Julio Gimenez}

Traducteur : Samuel Florin

\section{(2) OpenEdition}

\section{Journals}

Édition électronique

URL : http://journals.openedition.org/pratiques/1920

DOI : 10.4000/pratiques. 1920

ISSN : 2425-2042

\section{Éditeur}

Centre de recherche sur les médiations (CREM)

Édition imprimée

Date de publication : 15 juin 2012

Pagination : $35-50$

Référence électronique

Julio Gimenez, « Conceptualisations de l'esprit critique, des formes de validation et de l'effacement énonciatif dans l'écriture universitaire en sciences infirmièreset dans les formations de sages-femmes », Pratiques [En ligne], 153-154 | 2012, mis en ligne le 16 juin 2014, consulté le 03 mai 2019. URL http://journals.openedition.org/pratiques/1920 ; DOI : 10.4000/pratiques.1920 


\section{Conceptualisations de l'esprit critique, des formes de validation et de l'effacement énonciatif dans l'écriture universitaire en sciences infirmières ${ }^{(1)}$ et dans les formations de sages-femmes}

\section{Julio Gimenez}

Centre for English Language Education, School of Education, University of Notthingham, Royaume-Uni

\section{Traduit de l'anglais (G.B.) par Samuel Florin}

Étudiant en deuxième année du Master de Traduction « Métiers du Lexique et de la Traduction », Université Lille 3 (France)

\section{Introduction}

La place centrale qu'occupe l'écriture dans le parcours universitaire et son importance en termes de réussite scolaire ne font plus débat (Elander et al., 2006 ; Lillis \& Turner, 2001 ; Russel, 1991, 2001 ; Whitehead, 2002 ; Zhu, 2004 ; pour ne citer qu'eux). Il est également établi qu'il s'agit du principal outil dont disposent les étudiants pour donner forme à leur compréhension de la réalité sociale (ontologie) et faire preuve de leurs connaissances (épistémologie) (Gimenez, 2010), ainsi que ce sur quoi reposent les évaluations dans la plupart des universités du monde anglophone (Lillis \& Scott, 2007). Malgré la place centrale qu'elle occupe et les nombreuses études théoriques qui lui ont été consacrées, l'écriture est encore très souvent considérée comme une compétence à part que les étudiants doivent avoir acquise avant même d'entrer à l'université (Leki, 2003 ; Lillis \& Turner, 2001 ; Russell, 2001 ; Whitehead, 2002). Or, 1'ap-

(1) Note du traducteur : le terme «nursing » peut également se traduire par « formation des infirmières ». Le choix de traduction effectué ici rend compte d'un usage de plus en plus fréquent dans le domaine de la santé. 
proche qui consiste à voir dans l'écriture une compétence à part fait notamment l'impasse sur la spécificité disciplinaire que revêt la pratique de l'écriture dans l'enseignement supérieur (Bazerman, 2011). De même, cette approche conforte l'idée selon laquelle l'esprit critique, les formes de validation et l'effacement énonciatif, qui sont des propriétés caractéristiques de l'écriture disciplinaire dans l'enseignement supérieur, seraient transposables et indépendants du contexte dans lequel elle est produite, et pourraient donc aisément être déplacés d'un contexte à un autre. Cependant, ces dernières années, le caractère générique de l'écriture académique dans l'enseignement supérieur (Gimenez, 2008 ; Hyland, 2009) et le caractère transposable des propriétés en question (Jones, 2009 ; Moore, 2011) ont commencé à faire l'objet d'analyses plus poussées.

Cet article analyse donc la relation, en sciences infirmières et dans les formations de sages-femmes, entre les conceptualisations disciplinaires de l'esprit critique, des formes de validation et de l'effacement énonciatif, et l'écriture universitaire. Il s'appuie sur les données recueillies dans le cadre d'une étude de deux ans sur la nature et les dynamiques de l'écriture universitaire au sein des deux disciplines (Gimenez, 2008) et montre que la nature éminemment disciplinaire de ces propriétés contribue à la constitution d'écritures bien spécifiques, même dans ces deux formations parfois considérées comme très voisines.

Cet article s'attachera tout d'abord à passer en revue la recherche consacrée à la relation entre les positions épistémologiques et les propriétés disciplinaires qui a nourri l'étude mentionnée ci-dessus. Puis il décrira brièvement le contexte et la méthodologie de cette étude. Après quoi, il en présentera les résultats et les analysera. L'article s'achèvera sur une exploration des implications théoriques et pédagogiques des conclusions de l'étude.

\section{Positions épistémologiques et propriétés relatives à chaque discipline : l'état de la recherche.}

Il est établi de longue date que chaque discipline construit, expose et diffuse ses savoirs par des procédés qui lui sont propres. T. Becher a été l'un des premiers à mener une étude à grande échelle (Becher, 1989) visant à déterminer les traits caractéristiques prêtés au savoir dans plusieurs disciplines. Dans cette étude, il montre que les disciplines scientifiques voient le savoir comme une entité concrète, impersonnelle et exempte de valeurs, et qu'elles confèrent beaucoup d'importance aux objets matériels et à la présentation des informations. A contrario, les humanités ont tendance à estimer que le savoir est un concept abstrait, sujet à l'interprétation, et insistent davantage sur l'argumentation. T. Becher conclut que la manière dont une discipline construit, expose et diffuse ses savoirs a nécessairement une influence considérable sur la culture universitaire. Par conséquent, les positions épistémologiques disciplinaires occupent une place centrale, non seulement dans l'étude des cultures disciplinaires, mais également dans les recherches sur la conceptualisation de l'esprit critique, des formes de validation et de l'effacement énonciatif au sein de ces 
disciplines et dans leurs productions écrites. C'est ce qui a également permis de comprendre que les propriétés en question dépendent bien des contextes dans lesquels les textes sont produits. Ainsi, comme le montre A. Jones, des disciplines comme la médecine et l'histoire conceptualisent l'esprit critique de manière assez différente (Jones, 2009). Dans le domaine médical, l'esprit critique repose sur un bon raisonnement clinique et sur la compréhension de pratiques médicales et éthiques ancrées dans l'expérience, tandis que dans le domaine de l'histoire, il nécessite une analyse des données et l'identification des ambiguïtés et des idéologies qui caractérisent les événements historiques.

Des recherches antérieures ont analysé la conceptualisation de ces propriétés dans de nombreuses disciplines différentes : A. Jones, par exemple, s'est intéressée aux champs de l'économie et de l'histoire (Jones, 2007); puis elle s'est penchée sur l'histoire, la physique, l'économie, le droit et la médecine (Jones, 2008) ; quant aux recherches de T. J. Moore, elles ont été consacrées à la philosophie, à l'histoire et aux études littéraires (Moore, 2011). L'étude dont il est ici question s'est intéressée à deux disciplines sous-représentées dans le champ des recherches et qui, alors même qu'elles semblent souvent très proches, paraissent construire leurs savoirs différemment. Partant, il semble possible de supposer que les sciences infirmières et les formations de sages-femmes conceptualisent différemment l'esprit critique, les formes de validation et l'effacement énonciatif. Elles auraient donc deux conceptions différentes de l'écriture universitaire.

Selon les recherches menées à ce sujet, il semble que les sciences infirmières aient adopté une conception du savoir qui repose sur un modèle scientifique positiviste (Black, 2001 ; Segal, 2009 ; Spoel \& James, 2006). Elles semblent également plus enclines à utiliser des modèles linéaires de représentation du savoir (McCourt, 2005) pour analyser la relation entre santé et maladie (Vinson, 2000). A contrario, les formations de sages-femmes semblent considérer qu'il ne s'agit pas d'une notion figée, et privilégier le modèle constructiviste pour délimiter le champ de leurs savoirs (Hunter, 2008). De même, il apparaît généralement que les formations de sages-femmes tentent de combler la fracture cartésienne entre, d'une part, le raisonnement logique et les données empiriques, et de l'autre, la part d'intuition et de connaissances qui résulte des observations personnelles et de l'expérience professionnelle (Hunter, 2008 ; Lay, 2000). Les sciences infirmières, au contraire, s'abstiennent apparemment de recourir à l'intuition et cherchent à légitimer leurs savoirs en faisant appel aux connaissances empiriques, conceptuelles et cliniques (Kim, 2010 ; Vinson, 2000). P. Spoel et S. James montrent que les sciences infirmières ont tendance à suivre un modèle biomédical établi, tandis que les formations de sages-femmes privilégient un modèle d'accouchement centré sur la femme (Spoel \& James, 2006). M.M. Lay illustre également les différences entre les deux disciplines en mentionnant les tensions entre infirmiers, sages-femmes certifiées et sagesfemmes traditionnelles ${ }^{(2)}$ aux États-Unis, et les difficultés rencontrées par les

(2) Aux États-Unis, « les sages-femmes traditionnelles ("direct-entry midwifes" en anglais) entrent directement en formation de sages-femmes (ou commencent à pratiquer ce métier) sans formation préalable en sciences infirmières » (Lay, $2000: 2$ ). 
sages-femmes traditionnelles qui veulent préserver leur liberté d'exercice en refusant de suivre une formation en sciences infirmières (Lay, 2000).

Il faut toutefois comprendre que ces postures ontologiques et distinctions épistémologiques entre sciences infirmières et formations de sages-femmes sont relativement instables et extrêmement localisées. Malgré l'influence considérable du positivisme dans les sciences infirmières, cette discipline a commencé à adopter une approche plus relativiste de la profession au cours de ces dernières années (Black, 2001 ; Reed, 2006). Dans le même ordre d'idées, les positions épistémologiques qui ont cours au sein de ces disciplines subissent l'influence du contexte géographique et culturel. Aux États-Unis par exemple, la formation universitaire des sages-femmes certifiées et les sciences infirmières ne sont pas dissociées : la formation de sages-femmes est une spécialisation de deuxième cycle dans le cursus des sciences infirmières (Lay, 2000). Au Royaume-Uni, par contre, il s'agit d'une discipline à part entière, dotée de ses propres formations diplômantes de premier et deuxième cycles; elle a toutefois un organisme de contrôle en commun avec les sciences infirmières.

On peut donc formuler l'hypothèse suivante : les sciences infirmières et les formations de sages-femmes définissent leur discipline, construisent leurs savoirs et constituent leur culture disciplinaire de manières différentes, ce qui conditionne non seulement leur conceptualisation de l'esprit critique, des formes de validation et de l'effacement énonciatif, mais également leurs pratiques d'écriture. Ainsi, l'objectif de cette étude est de répondre aux questions suivantes :

1. Comment les positions épistémologiques des sciences infirmières et des formations de sages-femmes déterminent-elles la conceptualisation de l'esprit critique, des formes de validation et de l'effacement énonciatif dans le contexte envisagé ?

2. Dans quelle mesure cette conceptualisation conditionne-t-elle la pratique de l'écriture universitaire de premier cycle dans ces deux disciplines?

\section{Contexte et méthodologie de l'étude}

L'étude dont nous présentons ici les résultats s'est penchée sur la spécificité disciplinaire de l'écriture universitaire dans deux formations d'infirmiers et de sages-femmes de premier cycle d'une université de Londres, au RoyaumeUni : un BSc de Sciences infirmières (section Adultes) et un BSc de SagesFemmes. Il s'agit de deux formations de premier cycle en trois ans qui mènent respectivement à l'accréditation professionnelle en tant qu'Infirmier/Infirmière pour adultes et Sage-Femme. Plus précisément, l'objectif de l'étude était, entre autres, d'analyser la relation entre les positions épistémologiques de ces disciplines et la conceptualisation de l'esprit critique, des formes de validation et de l'effacement énonciatif, mais également l'influence de ces facteurs sur l'écriture universitaire de premier cycle.

Soixante-huit étudiants de sciences infirmières et soixante-sept étudiants en formation de sages-femmes, ainsi que quatre chargés d'enseignement, ont participé à l'étude. Les étudiants de ces formations présentaient des profils cultu- 
rels, ethniques, linguistiques et scolaires très variés, ce qui est typique de ce milieu (NMC, 2006). Parmi les participants, certains avaient commencé leur parcours universitaire juste après avoir obtenu leurs «A levels » (équivalent britannique du baccalauréat), d'autres après avoir suivi des « Access courses » (formation préparant au premier cycle universitaire), et d'autres encore après avoir obtenu un « Advanced Vocational Certificate of Education » (formation professionnelle du secondaire).

Les données de l'étude ont été recueillies grâce à un questionnaire, à dix entretiens très détaillés, à deux focus groupes - un pour chaque discipline - et à des travaux écrits réalisés par les étudiants. Le questionnaire comportait deux parties : la première concernait les étudiants et leur formation, et la seconde leurs opinions et leurs pratiques de l'écriture universitaire. Les questions portaient notamment sur les genres auxquels ils étaient le plus confrontés durant leur formation, sur le niveau de difficulté que présentaient selon eux ces genres, sur la manière dont ils s'y prenaient pour les écrire, ainsi que sur l'esprit critique, les formes de validation et l'effacement énonciatif. Les étudiants devaient choisir leurs réponses parmi une liste de réponses pré-établies, mais ils avaient la possibilité d'utiliser la catégorie « Autres » pour développer leurs réponses, ou de remplir une section intitulée «Vos remarques ${ }^{(3)}$ », ce qu'ils ont fait très souvent.

Ce sont des entretiens semi directifs qui ont été employés afin de recueillir des points de vue plus complets sur la spécificité disciplinaire de l'écriture universitaire. Quatre chargés d'enseignement et six étudiants - trois infirmiers et trois sages-femmes - y ont participé. La liste des questions posées durant les entretiens est reproduite dans l'annexe. Chaque focus groupe était composé de six étudiants qui se voyaient poser une série de questions plus ou moins structurées, conçues à partir d'une analyse préalable des données recueillies grâce au questionnaire et aux entretiens. Les étudiants participant à l'étude devaient également choisir des extraits des productions écrites qu'ils considéraient représentatives des exigences de leur formation en termes d'écriture. Le tableau 1 (page suivante) répertorie la liste des 28 productions écrites choisies par les étudiants.

Les réponses au questionnaire ont été traitées à l'aide du logiciel SPSS, et les entretiens, ainsi que les données des groupes-témoins, ont été transcrites et codées avec le progiciel AQUAD 5, utilisé pour l'analyse de données qualitatives. Une fois les données transcrites, nous avons procédé à l'analyse des transcriptions afin de déterminer les grands concepts qui s'en dégageaient et les premières catégories de codage. Nous avons pour ce faire utilisé la démarche établie par A. Strauss (Strauss, $1987: 27$ ) : ainsi, on tente tout d'abord de réaliser " un codage ouvert des données » afin de trouver les grands concepts (par exemple, l'esprit critique). Ensuite, le codage est affiné, jusqu'à l'obtention d'une « liste de codes » pour chacun des concepts (par exemple, l'usage critique des sources). Enfin, des «liens» (p. 33) sont établis entre les codes ainsi affinés afin d'établir entre eux une relation significative (par exemple, l'usage critique des sources + les sciences infirmières). La transcription des données est restée

(3) On trouvera une description plus détaillée des résultats dans Gimenez, 2008. 


\begin{tabular}{|c|c|c|}
\hline Texte & Discipline & Quantité \\
\hline \multirow[t]{2}{*}{ Essais $\quad{\text { essays })^{(4)}}^{(4)}$} & Sciences infirmières & 4 \\
\hline & Formation de sages-femmes & 2 \\
\hline Réflexion sur les soins & Formation de sages-femmes & 4 \\
\hline \multirow[t]{2}{*}{ Projet de mémoire } & Sciences infirmières & 3 \\
\hline & Formation de sages-femmes & 3 \\
\hline Planifications des soins & Sciences infirmières & 3 \\
\hline Études de cas & Sciences infirmières & 2 \\
\hline \multirow[t]{2}{*}{ Textes réflexifs } & Sciences infirmières & 3 \\
\hline & Formation de sages-femmes & 4 \\
\hline TOTAL & & 28 \\
\hline
\end{tabular}

Tableau 1. Textes choisis par les étudiants

aussi simple que possible : le texte souligné représente des mots prononcés sur un ton appuyé, le [texte] entre crochets indique un chevauchement, et les points de suspension entre parenthèses (...) indiquent des sections inaudibles ou incompréhensibles.

\section{Conceptualisation des propriétés et spécificité de l'écriture dans chaque discipline : exploration des résultats}

Cette partie de l'article présente les résultats de l'analyse de la relation entre la conceptualisation des propriétés de l'écriture dans ces deux disciplines et son influence sur l'écriture universitaire. Dans un premier temps, on s'intéressera à l'esprit critique, aux formes de validation et à l'effacement énonciatif en sciences infirmières et en formation de sages-femmes, avant de se pencher sur la façon dont ces propriétés se traduisent dans l'écriture.

\subsection{Esprit critique, formes de validation et effacement énonciatif en sciences infirmières}

Les positions épistémologiques des sciences infirmières et des formations de sages-femmes décrites ci-dessus conditionnent la conceptualisation de pro-

(4) Note du traducteur : comme dans les autres articles de cette livraison, l'anglais essay est traduit par le terme plus proche d'essai, et non par sa traduction ordinaire de dissertation. Pour cette raison, nous le maintenons en italique tout au long de cet article. 
priétés comme l'esprit critique, les formes de validation et l'effacement énonciatif. Ces conceptualisations, en retour, façonnent la manière dont chaque discipline construit ses pratiques d'écriture, comme nous le verrons ci-dessous dans le 4.3.

En sciences infirmières, la notion d'esprit critique est étroitement liée à la " résolution de problèmes », sans doute en raison de la manière dont ce milieu construit ses savoirs, ainsi que des modèles de représentation du savoir qu'il applique pour expliquer la relation entre santé et maladie (Kim, 2010 ; Vinson, 2000). Dans l'ensemble, en sciences infirmières, "être critique » revient à être capable d'analyser, de décrire et d'expliquer la relation entre ces deux états. Cette définition de l'esprit critique ressort très clairement des données de l'étude, comme le montrent les citations suivantes :

c'est dur de sortir de la dichotomie santé-maladie, parce qu'en général on se sert de l'une pour définir l'autre... bien sûr on a fait beaucoup de chemin depuis la première définition de la santé de l'OMS où on trouve, vous savez, des mots comme "un état de complet bien-être" et euh... maintenant on voit ça de façon plus réaliste si vous voulez... mais vous savez, c'est toujours très pratique de voir les liens entre santé et maladie... et c'est pour cette raison qu'on attend des étudiants qu'ils soient critiques par rapport à ça... (Mark ${ }^{(5)}$, chargé d'enseignement en sciences infirmières)

Oui, je pense... quand on est critique par rapport à la façon dont la santé a été définie, et qu'on sait que dans certains cas elle est toujours définie comme ça, on se demande comment on peut aider les patients qui souffrent d'une maladie donnée à aller mieux, vous savez... (Rita, étudiante en sciences infirmières, à propos de son essai sur les définitions de la santé)

La conceptualisation des formes de validation semble également dépendre très étroitement de l'épistémologie de chaque discipline. Il ressort de la présente étude que les sciences infirmières préfèrent les essais contrôlés randomisés (ECR) à d'autres formes de validation. Même si certains critiquent la prédominance des ECR parmi les "principales sources scientifiques" de disciplines comme les sciences infirmières (voir par exemple Black, 2001 ; McCourt, 2005), les ECR ont toutefois leur préférence parce qu'ils sont fiables et permettent de minimiser le risque de biais, ainsi que de procéder à des comparaisons équitables (Black, 2001). Lors de l'étude, de nombreux participants mentionnent également cette préférence :

Je crois qu'en sciences infirmières, c'est important que vos sources viennent de ce qu'on appelle des sources scientifiques : vous savez, les revues internationales, les rapports du NHS, les ECR et des choses comme ça... que vous deviez vous servir de plusieurs sources différentes, ça aussi c'est vrai, mais je crois qu'en majorité ça devrait être des sources de ce type... (Anthony, étudiant en sciences infirmières, à propos des travaux écrits)

oui, vous savez, au semestre dernier par exemple on a eu beaucoup d'informa-

(5) Tous les noms ont été remplacés par des pseudonymes. 
tions sur comment faire de la recherche et à quel point c'est important qu'on y participe tous, vous savez, lire des articles de recherche c'est important bien sûr, c'est là que vous trouvez toutes vos formes de validation, mais faire de la recherche c'est tout aussi important, eh bien, pour apporter une sorte de contribution... de contribution scientifique à la discipline (Carol, étudiante en sciences infirmières)

et plus vos formes de validation sont rigoureuses et scientifiques, plus les conclusions que vous pouvez en tirer sont fortes, et [que]

Enquêteur : [et] quelles sont les formes de validation qui seraient considérées rigoureuses ou... scientifiques?

Eh bien... celles qui s'appuient sur des sources officielles, comme les rapports du NHS, ou du NMC ou du NICE ${ }^{(6)}$, et bien sûr les ECR... (Alex, étudiant en sciences infirmières)

En ce qui concerne l'effacement énonciatif, il semble que l'on observe des normes différentes d'une discipline à une autre (Harwood, 2005 ; Hyland, 2002). Cependant, dans ce domaine, on constate aussi bien des différences que des points communs entre les sciences infirmières et les formations de sagesfemmes. Dans ces deux disciplines, on recommande aux étudiants d'utiliser un style impersonnel, principalement afin d'éviter que l'attention du lecteur ne se porte sur l'auteur du texte au lieu du texte lui-même. Pour la plupart des étudiants, cela entraîne des difficultés linguistiques et identitaires : il leur apparaît souvent difficile de ne pas être présents dans leurs textes sur le plan linguistique; difficile aussi d'accepter de renoncer à ce qui leur est propre, ce qui les identifie, ce qui fait leur autorité à travers leurs écrits. Comme le disent deux étudiantes :

Je ne sais jamais quoi écrire, vous savez... C'est comme parler de quelqu'un d'autre, écrire à votre sujet comme si vous n'y étiez pas, c'est tellement bizarre... quand je lis ce que j'ai écrit, vous savez, j'ai l'impression que c'est le travail de quelqu'un d'autre. (Cynthia, étudiante en sciences infirmières)

Comment voulez-vous parler de l'étudiante sage-femme alors qu'en fait c'est de vous-même que vous parlez? (Vera, étudiante en formation de sage-femme)

Les sciences infirmières et les formations de sages-femmes, toutefois, divergent quant à l'importance qu'elles accordent à l'effacement énonciatif dans les travaux écrits. En sciences infirmières, l'effacement énonciatif participe de l'objectivité, et il est donc prisé dans la quasi-totalité des textes :

Vous savez, il y a cette tradition de ne pas utiliser de formes contractées ou de pronoms personnels dans les travaux écrits comme les essais, par exemple, alors on dit aux étudiants d'éviter de s'en servir dans leurs comptes rendus (Mark, chargé d'enseignement en sciences infirmières)

(6) Note du traducteur : le NHS («National Health Service»), NMC (« Nursing and Midwifery Council») et le NICE («National Institute for Health and Clinical Excellence ») sont trois organisations officielles du système de santé britannique. 


\subsection{Esprit critique, formes de validation et effacement énonciatif dans les formations de sages-femmes.}

Les formations de sages-femmes diffèrent passablement des sciences infirmières quant à leur conception de l'esprit critique. Dans la lignée de l'approche constructiviste adoptée par les formations de sages-femmes, l'esprit critique est défini comme une propriété à multiples facettes, qui fait intervenir la résolution de problèmes, mais également la réflexion critique, la bonne compréhension des idéologies et des évolutions sociales, ainsi que la critique des théories et pratiques des sages-femmes dans leurs contextes socio-politiques, historiques et culturels (Siddiqui, 2005). Cette conceptualisation de l'esprit critique est avérée dans les travaux écrits fournis par certains des étudiants et dans les entretiens avec les deux chargés d'enseignement.

La douleur ressentie par une femme qui accouche peut changer d'un stade du travail à un autre. Par exemple, durant le premier stade, la douleur vient des récepteurs sensoriels appelés nocicepteurs, situés dans l'utérus et le périnée. Durant le deuxième stade, la douleur ressentie émane des deuxième, troisième et quatrième segments sacrés, qui peuvent être anesthésiés par le biais d'une péridurale (Leong et al., 2000). Cependant, les avis concernant le recours à la péridurale sont partagés : certaines études la recommandent (par exemple Crosby, 1990 ; Simkin, 1997 ; Stem, 1997) et d'autres s'y opposent (notamment Halpern et al., 1998 ; Howell, 1999 ; Robinson et al., 1999). De même, le recours à la péridurale est critiqué parmi les sages-femmes qui privilégient les naissances normales aux naissances accélérées et s'opposent aux péridurales durant le deuxième stade de l'accouchement (O'Driscoll \& Meagher, 1989). L'augmentation récente du nombre de femmes qui demandent elles-mêmes des péridurales lors des rendez-vous prénatals souligne également la nécessité d'informer les femmes des avantages et des inconvénients que présente le recours aux péridurales pendant le travail et l'accouchement. (Martha, étudiante sage-femme, dans un essai sur le recours aux péridurales pendant l'accouchement)

(...) les étudiantes sages-femmes doivent acquérir tout un ensemble de connaissances complexes. Pour acquérir les savoirs dont une sage-femme a besoin il ne suffit pas de suivre une ligne droite, ça ne fait pas seulement intervenir l'anatomie et la physiologie du corps humain vous savez, comme le corps et le déroulement de la maternité, l'utérus, le placenta et tout ça, la formation de sages-femmes fait intervenir d'autres aspects qui auront aussi des conséquences sur la façon dont l'accouchement sera vécu, vous savez: la femme est-elle primigeste? quelle position a-t-elle choisie ? comment voit-elle l'accouchement ? quelles sont ses croyances sociales et culturelles par rapport à l'accouchement ? aux soins postnatals ? à l'allaitement? C'est important que la sage-femme sache tout cela... (Adèle, chargée d'enseignement en formation de sages-femmes)

Quant aux formes de validation, elles sont conceptualisées dans les formations de sages-femmes comme faisant intervenir à la fois les résultats « d'enquêtes scientifiques, objectives et rigoureuses » $(\mathrm{DoH}, 1996)$ et l'expérience clinique, ainsi que les connaissances concrètes, acquises grâce à l'observation et aux expériences personnelles (Fullbrook, 2004). C'est ce que montrent les 
deux citations suivantes, extraites des entretiens avec les étudiant(e)s sagesfemmes :

...c'est ce que je fais dans ce texte, vous voyez... Je crois que c'est important de trouver un équilibre entre les recherches scientifiques, comme les revues et les rapports du NICE par exemple, mais aussi vos propres expériences, grâce aux stages et à l'observation... (Martha, étudiante sage-femme, à propos de l'écriture en formation de sages-femmes)

très important, d'après moi, mais pas plus que ce qu'on apprend durant les stages cliniques, je crois que l'observation est aussi importante ; en classe et pendant les stages, vous savez, l'accent est mis sur le fait qu'on doit développer nos compétences d'observation pour comprendre et intégrer la situation de façon globale... (Sally, étudiante sage-femme)

Nous avons vu que les sciences infirmières et les formations de sages-femmes ont certains traits communs concernant l'effacement énonciatif dans l'écriture universitaire. Mais en formation de sages-femmes, contrairement aux sciences infirmières, le style impersonnel ne vaut que pour certains types de textes, en particulier ceux qui ont trait à la pratique professionnelle :

Oui, c'est vrai, on encourage les étudiants à éviter de se servir de pronoms personnels comme je ou nous, mais c'est seulement dans les textes où l'attention des lecteurs doit se porter sur ce qui est dit plutôt que sur la personne qui le dit... dans les textes réflexifs, par exemple, on ne leur conseillerait jamais d'éviter le je... (Adèle, chargée d'enseignement en formation de sages-femmes)

\subsection{Esprit critique, formes de validation et effacement énonciatif dans l'écriture universitaire}

Les résultats exposés ci-dessus montrent que la façon dont les disciplines envisagent les savoirs et la construction de savoirs joue un rôle fondamental dans la manière dont une discipline conceptualise des propriétés comme l'esprit critique, les formes de validation et l'effacement énonciatif : elles dépendent donc d'un certain contexte, et ne sont ni génériques, ni transposables. Là où les sciences infirmières voient surtout l'esprit critique comme une « résolution de problèmes ", les formations de sages-femmes le conceptualisent comme « un raisonnement contextuel et relativiste » (Parry, 1998). Lorsque les étudiants de sciences infirmières doivent faire montre de leurs compétences critiques à l'écrit, par conséquent, ils doivent synthétiser et comparer les données de recherche issues de sources diverses, qui sont tenues de respecter le critère « de scientificité» dicté par leur discipline. Les étudiants en formation de sages-femmes sont pour leur part confrontés à une tâche plus complexe. En effet, ils doivent non seulement synthétiser et comparer les données scientifiques, mais également prouver qu'ils sont capables d'intégrer plusieurs types de données issues de sources diverses, dont certaines ne respectent pas nécessairement le critère de scientificité requis en sciences infirmières.

Tandis que les étudiants en sciences infirmières sont généralement tenus de montrer que leurs travaux écrits s'appuient sur des données scientifiques sélec- 
tionnées de façon rigoureuse, des définitions bien établies et des théories de production de savoirs, les étudiants en formation de sages-femmes doivent habituellement combiner les conclusions scientifiques des travaux de recherche et ce qu'ils ont appris au cours de leurs observations et de leurs expériences cliniques, afin de faire le lien avec des problématiques spécifiques relatives à la pratique professionnelle.

Il en va de même pour la conceptualisation disciplinaire des formes de validation. Dans le cadre du modèle positiviste qui prévaut en sciences infirmières, on préfère les données scientifiques à d'autres types de données; dans le cadre du modèle constructiviste, plus répandu en formation de sages-femmes, les formes de validation semblent renvoyer à un concept plus multiforme : cela demande aux auteurs de concilier une gamme de possibilités plus vaste. Ainsi, en sciences infirmières, on considère souvent que les formes de validation sont " indépendantes du contexte » (Lomas et al., 2005) et que les sources peuvent être organisées hiérarchiquement selon leur valeur scientifique : les ECR sont au sommet de la hiérarchie, et les avis des spécialistes en bas de l'échelle. Les formations de sages-femmes, elles, abordent les formes de validation comme une propriété « relative au contexte».

Ces différentes conceptions des formes de validation se reflètent également dans ce qu'on attend des étudiants lorsqu'ils rendent des travaux universitaires écrits, comme on peut le constater à la lecture des deux extraits suivants, tirés de textes fournis par les étudiants qui participaient à l'étude :

Faire baisser la tension artérielle des patients qui ont une tension trop élevée est une tâche très difficile à laquelle les infirmiers travaillant dans le milieu éducatif de la santé sont toujours confrontés aujourd'hui. Les conseils donnés aux patients qui vont à l'infirmerie ne sont pas toujours couronnés de succès, malgré le fait que les infirmiers ne se contentent pas de donner des conseils, mais distribuent aussi divers documents (par exemple, des dépliants). Les interventions de type comportemental, par contre, rencontrent plus de succès. Un nombre considérable d'études très importantes s'appuyant sur des essais contrôlés randomisés, comme celle menée par Appel et al. (2003), pour mesurer l'efficacité des interventions comportementales (par exemple, ELR et DASH ${ }^{(7)}$ ) chez les sujets ayant une tension trop élevée, ont démontré la supériorité de ces interventions par rapport aux conseils des infirmiers. (Joy, étudiante en sciences infirmières, dans un essai sur l'éducation de la santé)

Contrairement aux soins médicaux, dans le cadre desquels un obstétricien ou un médecin prennent en charge une femme lors de son accouchement, les soins procurés par les sages-femmes reposent sur des soins réguliers et continus, qui sont procurés avant la naissance, pendant l'accouchement, et après la naissance (Robinson et al., 1999). Ils mettent en lumière le fait qu'une femme peut accoucher naturellement avec un minimum d'interventions médicales. Ils soulignent également l'importance des soins apportés à la femme et au bébé ; l'importance

(7) Note du traducteur : les ELR («Established Lifestyle Recommendations », Recommandations en vigueur concernant l'hygiène de vie) et les DASH (« Dietary Approaches to Stop Hypertension », Régimes alimentaires pour lutter contre l'hypertension) sont deux types d'interventions comportementales. 
aussi de leur origine ethnique, des besoins spécifiques à leur origine sociale et, dans certains cas, de leurs croyances religieuses (notes sur les stages cliniques). (Stelle, étudiante sage-femme, réflexion sur les soins)

Même si, comme établi dans la partie précédente, les deux disciplines attendent généralement des étudiants qu'ils écrivent de façon impersonnelle, les étudiants en sciences infirmières doivent adopter un style impersonnel dans presque toutes leurs productions écrites, et même dans leurs textes réflexifs (voir l'extrait de l'entretien avec Cynthia ci-dessus). En formation de sagesfemmes, par contre, le style impersonnel est décrit de façon plus relative : il est recommandé dans les productions écrites consacrées à la pratique professionnelle, mais on accepte généralement un style plus personnel dans les textes réflexifs.

Par conséquent, il serait bon d'exposer clairement aux étudiants la spécificité disciplinaire et épistémologique de ces propriétés au lieu de les présenter comme des caractéristiques transposables. De même, il faudrait leur faire comprendre le rôle de ces propriétés, qui participent à la spécificité disciplinaire des pratiques d'écriture à l'université : c'est ce que s'attache à démontrer la partie suivante.

\section{Analyse des implications théoriques et pédagogiques}

L'analyse des relations entre les positions épistémologiques disciplinaires, les propriétés de l'écriture universitaire (qui dépendent du contexte) et les pratiques d'écriture (qui diffèrent selon les disciplines), peut ouvrir des perspectives théoriques et pédagogiques importantes.

En ce qui concerne la portée théorique, il est important d'étudier l'influence des positions épistémologiques disciplinaires sur la façon dont les disciplines conceptualisent les propriétés de l'écriture universitaire - notamment l'esprit critique, les formes de validation et l'effacement énonciatif - que l'on a longtemps considérées indépendantes du contexte, et par conséquent transposables. Cependant, des études récentes ont démontré que ces propriétés dépendent en réalité du contexte, et diffèrent donc d'une discipline à l'autre (Jones, 2009 ; Moore, 2011). Notre étude a établi que c'était également le cas dans des disciplines que l'on considère souvent très proches comme les sciences infirmières et les formations de sages-femmes.

Cette conclusion est également d'importance quant à la théorisation de l'écriture disciplinaire à l'université. L'esprit critique, les formes de validation et l'effacement énonciatif sont des propriétés fondamentales de l'écriture universitaire dans l'enseignement supérieur et jouent donc un rôle important dans la définition des croyances et des attentes de chaque discipline en matière d'écriture. Ces croyances et ces attentes, qui participent des pratiques disciplinaires, restent parfois "invisibles" dans certaines conceptions de l'écriture universitaire (par exemple, celle de l'EAP, «English for Academic Purposes », formation à l'écriture universitaire qui prend surtout le texte en compte) et peu- 
vent ainsi devenir des obstacles insurmontables pour les étudiants (Gimenez, 2010).

De même, il faudrait mettre en place une approche pédagogique des spécificités disciplinaires de l'écriture universitaire afin de sensibiliser les étudiants au caractère relatif de ces propriétés et à leur rôle. Ces éléments sont très peu abordés et encore moins enseignés : les difficultés que rencontrent les étudiants lorsqu'ils doivent produire des textes respectant cette spécificité disciplinaire tendent à passer inaperçues car elles sont qualifiées de problèmes linguistiques et rédactionnels (Lillis et Scott, 2007 ; McCune, 2004). Toutefois, pour que les étudiants puissent produire des textes disciplinaires de qualité et participer activement à la construction des savoirs de leur discipline, les propriétés de l'écriture universitaire (notamment l'esprit critique, les formes de validation et l'effacement énonciatif) devraient être clairement enseignées dans ces disciplines. De plus, leur rôle dans la définition de la spécificité disciplinaire des pratiques d'écriture devrait faire l'objet de recherches précises.

\section{Conclusion}

À partir des données recueillies grâce à une étude de deux ans sur la nature et les dynamiques de l'écriture universitaire en sciences infirmières et en formation de sages-femmes, cet article a analysé la relation entre les conceptualisations disciplinaires de l'esprit critique, des formes de validation et de l'effacement énonciatif, et l'écriture universitaire au sein de ces deux disciplines.

La conclusion de cet article est que d'importantes perspectives théoriques et pédagogiques s'ouvrent grâce à l'analyse de la relation entre les positions épistémologiques disciplinaires, les propriétés de l'écriture universitaire (qui dépendent du contexte) et l'écriture disciplinaire. En ce qui concerne la portée théorique, l'objet de l'étude était de montrer que l'analyse des positions épistémologiques disciplinaires est primordiale : en effet, elle permet de comprendre leur rôle dans la conceptualisation des propriétés de l'écriture universitaire. Dans le même ordre d'idées, c'est en examinant les différences de conceptualisation de ces propriétés d'une discipline à l'autre que l'on peut comprendre les relations entre le contexte et les propriétés de l'écriture universitaire. Cette démarche, ensuite, permettrait de souligner plus clairement le rôle central des propriétés que sont l'esprit critique, les formes de validation et l'effacement énonciatif dans les pratiques d'écriture académiques disciplinaires.

Quant à la portée pédagogique, notre étude a mis en lumière la nécessité de sensibiliser plus explicitement les étudiants au fait que ces propriétés dépendent du contexte, aux liens qu'elles entretiennent avec les positions épistémologiques en vigueur dans leur discipline, et à la manière dont tout cela devrait se répercuter sur leurs productions écrites. En conséquence de quoi, progressivement, les nouveaux membres d'une discipline pourraient accéder plus aisément à son corpus et, bien mieux, ne se trouveraient plus relégués à la périphérie de leur culture disciplinaire. 


\title{
Remerciements
}

Je tiens à exprimer ma reconnaissance aux étudiants et aux chargés d'enseignement qui ont participé à cette étude pour leurs contributions et leur dévouement, ainsi que pour le temps qu'ils lui ont consacré. Je remercie également Paula Carlino et David R. Russell pour les remarques qu'ils m'ont apportées à la lecture des premières versions de cet article. Toutes les erreurs qui subsistent sont de ma seule responsabilité.

\begin{abstract}
Annexe
Liste des questions posées lors des entretiens semi directifs (il s'agit de questions générales sur lesquelles l'enquêteur s'appuyait pour approfondir à partir des réponses fournies par les participants) :

1. description de l'écriture universitaire en sciences infirmières/formations de sages-femmes

2. quelles sont les tâches les plus difficiles auxquelles les participants/les étudiants sont généralement confrontés?

3. doit-on faire preuve d'esprit critique dans les travaux écrits des sciences infirmières/formations de sages-femmes?

4. comment faire preuve d'esprit critique dans les travaux écrits des sciences infirmières/formations de sages-femmes?

5. le rôle des formes de validation dans les travaux écrits de sciences infirmières/formations de sages-femmes

6. qu'appelle-t-on «formes de validation » dans les travaux écrits de sciences infirmières/formations de sages-femmes?

7. l'utilisation de pronoms personnels (comme « je » ou «nous ») dans les travaux écrits de sciences infirmières/formations de sages-femmes
\end{abstract}




\section{Références}

BAZERMAN, C. (2011) : "The Disciplined Interdisciplinarity of Writing Studies », Research in the Teaching of English, vol. 46.1, pp. 8-21.

BECHER, T. (1989) : Academic Tribes and Territories: Intellectual Enquiry and the Cultures of Disciplines, Milton Keynes, Open University Press.

BLACK, N. (2001) : «Evidence Based Policy : Proceed with Care », British Medical Journal, vol. 323.4, pp. 275-279.

DePartment OF HeAlth (1996) : Promoting Clinical Effectiveness : A Framework for Action in and through the NHS, Londres, Department of Health.

Elander, J., Harrington, K., Norton, L., Robinson, H. \& Reddy, P. (2006) : «Complex Skills and Academic Writing : A Review of Evidence about the Types of Learning Required to Meet Core Assessment Criteria », Assessment and Evaluation in Higher Education, vol 31.1, pp. 71-90.

FUlLBROOK, P. (2004) : «Realizing Advanced Nursing Practice through Reflection », British Association of Critical Care Nurses, vol. 9.6, pp. 255-256.

GimenEZ, J. (2008) : «Beyond the Academic Essay : Discipline-specific Writing in Nursing and Midwifery », Journal of English for Academic Purposes, vol 7.3, pp. 151-164.

- (2010) : «Academic Writing in the Disciplines : Practices in Nursing, Midwifery and Social Work », in M. F. Ruiz-Garrido, J. C. Palmer-Silveira \& I. Fortanet-Gomez, ed., English for Professional and Academic Purposes, Amsterdam, Rodopi, pp. 197-211.

HaRwoOD, N. (2005) : " "I Hoped to Counteract the Memory Problem, but I Made no Impact Whatsoever" : Discussing Methods in Computing Science Using », English for Specific Purposes, vol. 23.4, pp. 243-267.

Hunter, L. P. (2008) : «A Hermeneutic Phenomenological Analysis of Midwives' Ways of Knowing during Childbirth », Midwifery, vol 24.4, pp. 405415.

HYLAND, K. (2002) : « Specificity Revisited: How far Should we Go Now ? », English for Specific Purposes, vol 21.4, pp. 385-395.

- (2009) : Academic Discourse, London, Continuum.

JONES, A. (2007) : «Re-disciplining Generic Attribute», Article présenté dans le cadre de la conférence EARLI, Budapest, 28 août $-1^{\text {er }}$ septembre.

- (2008) : " Generic Attributes as Espoused Theory : The Importance of Context », Higher Education, vol. 58.2, pp. 175-191.

- (2009) : «Redisciplining Generic Attributes : The Disciplinary Context in Focus », Studies in Higher Education, vol. 34.1, pp. 85-100.

KIM, H. S. (2010) : The Nature of Theoretical Thinking in Nursing, New York, Springer Publishing Company.

LAY, M. M. (2000) : The Rhetoric of Midwifery: Gender, Knowledge, and Power, New Jersey, Rutgers University Press.

LEKI, I. (2003) : « Living through College Literacy : Nursing in a Second Language », Written Communication, vol. 20.1, pp. 81-98.

LILLIS, T., Scott, M. (2007) : «Defining Academic Literacies Research : Issues of Epistemology, Ideology and Strategy », Journal of Applied Linguistics, vol1 4.1, pp. 5-32.

LILLIS, T., TURNER, J. (2001) : « Student Writing in Higher Education : Contem- 
porary Confusion, Traditional Concerns », Teaching in Higher Education, vol. 6.1, pp. 57-68.

Lomas, J., Culyer, T., McCutcheon, C., McAuley, L. \& LAW, S. (2005) : Conceptualizing and Combining Evidence for Health System Guidance, Ottawa, Canada, Canadian Health Services Research Foundation, http ://www.chsrf.ca/other_documents/pdf/evidence_e.pdf, (Consulté le 3 septembre 2010)

MCCOURT, C. (2005) : "Research and Theory for Nursing and Midwifery : Rethinking the Nature of Evidence », Worldviews on Evidence-Based Nursing, vol. 2.2, pp. 75-83.

MCCunE, V. (2004) : « Development of First-year Students' Conceptions of Essay Writing », Higher Education, vol. 47.2, pp. 257-282.

Moore, T. J. (2011) : «Critical Thinking and Disciplinary Thinking : A Continuing Debate ", Higher Education Research \& Development, vol. 30.3, pp. 261-274.

NURSING AND MIDWIFERY COUNCIL (NMC) (2006) : Becoming a Midwife in the UK, (Consulté le 16 novembre 2006)

PARRY, S. (1998) : "Disciplinary discourse in doctoral theses », Higher Education, vol. 36.3, pp. 273-299.

REED, P. G. (2006) : « The Practice Turn in Nursing Epistemology », Nursing Science Quarterly, vol. 19.2, pp. 36-38.

RUSSELL, D. R. (1991) : Writing in the Academic Disciplines, 1870-1990 : A Curricular History. Carbondale, Southern Illinois University Press.

- (2001): «Where Do the Naturalistic Studies of WAC/WID Point? A Research Review », in S. H. McLeod, E. Miragia, M. Soven \& C. Thaiss, ed., WAC for the New Millennium: Strategies for Continuing Writing-Acrossthe-Curriculum Programs, Urbana, NCTE, pp. 259-98.

SEGAL, J. Z. (2009) : "Rhetoric of Health and Medicine », in A. A. Lunsford, K. H. Wilson \& R. A. Eberly, ed., The Sage Handbook of Rhetorical Studies, Thousand Oaks, Calif., Sage, pp. 227-246.

SIDDIQUI, J. (2005) : « The Role of Knowledge in Midwifery Decision Making », in M. Raynor, J. Marshall \& A. Sullivan, ed., Decision Making in Midwifery Practice, London, Elsevier, pp. 23-36.

SPOEL, P. \& JAMES, S. (2006) : « Concept Analysis : Using an Academic Nursing Genre for Writing Instruction in Nursing ", in B. Heifferon \& S. C. Brown, ed., Rhetoric of Healthcare : Essays toward a New Disciplinary Inquiry, Cresskill, NJ, Hampton Press, pp. 243-263.

Strauss, A. L. (1987) : Qualitative Analysis for Social Scientists, Cambridge, Cambridge University Press.

Vinson, J. A. (2000) : «Nursing's Epistemology Revisited in Relation to Professional Education Competencies », Journal of Professional Nursing, vol. 16.1, pp. 39-46.

WhiteheAD, D. (2002) : «The Academic Writing Experiences of a Group of Student Nurses : A Phenomenological Study », Journal of Advanced Nursing, vol. 38.5, pp. 498-506.

ZHU, W. (2004) : «Writing in Business Courses : An Analysis of Assignment Types, their Characteristics, and Required Skills », English for Specific Purposes, pp. 111-135. 